UW/PT-92-19

ANL-HEP-PR-92-51

UR-1287

ER-40685-736

\title{
On the Analytic Structure of the Self-Energy for Massive Gauge Bosons at Finite Temperature
}

\author{
Peter Arnold \\ Stamatis Vokos \\ Department of Physics, FM-15, \\ University of Washington, \\ Seattle, WA 98195 \\ Paulo Bedaque \\ Ashok Das \\ Department of Physics and Astronomy, \\ University of Rochester, \\ Rochester, NY 14627
}

\begin{abstract}
We show that the one-loop self-energy at finite temperature has a unique limit as the external momentum $p_{\mu} \rightarrow 0$ if the loop involves propagators with distinct masses. This naturally arises in theories involving particles with different masses as is demonstrated for a toy model of two scalars as well as in a $U(1)$ Higgs theory. We show that, in spontaneously broken gauge theories, this observation nonetheless does not affect the difference between the Debye and plasmon masses, which are often thought of as the $\left(p_{0}=0, \vec{p} \rightarrow 0\right)$ and $\left(p_{0} \rightarrow 0, \vec{p}=0\right)$ limits of the self-energy.
\end{abstract}

October 1992 


\section{Introduction}

In finite temperature field theory, the existence of an additional four-vector, namely the four-velocity of the plasma, allows one to construct two independent Lorentz scalars on which the Green's functions can depend,

$$
\begin{aligned}
& \omega=P \cdot u \\
& k=\sqrt{\left[(P \cdot u)^{2}-P^{2}\right]}
\end{aligned}
$$

where $u^{\mu}$ is the four-velocity of the plasma and $P^{\mu}=\left(p^{0}, \vec{p}\right)$ is the four-momentum of any particle. In the rest-frame of the fluid, these scalars reduce to $p^{0}$ and $p=|\vec{p}|$ respectively.

In particular, this separate dependence of polarization tensors and self-energies, allows one to take the limits $p^{0} \rightarrow 0$ and $p \rightarrow 0$ in different orders and, in general, one expects that the limits need not commute. In fact, it has been shown that in the case of hot QCD近, and self-interacting scalars 23,3, the two limits do not indeed commute. In this paper, however, we will show that there exist contributions to the one-loop self-energy of a massive gauge boson in a spontaneously broken gauge theory which possess a unique limit as $p$ and $p^{0}$ tend to zero, as long as the particles propagating in the loop have different masses. As we shall show, however, for the purposes of computing physical quantities, such as poles of particle propagators, the usual approximation which uses the non-commuting limits is perfectly adequate.

The outline of the paper is as follows. In Section 2, we study the contribution to the

self-energy of a scalar field $\phi_{1}$ through its coupling to another scalar $\phi_{2}, \mathcal{L}_{I}=-\frac{\lambda}{2} \phi_{1}^{2} \phi_{2}$, where the two fields have different masses. In Section 3, we analyze the polarization tensor of the massive photon in a spontaneously broken $U(1)$ theory, where the Higgs and the photon have different masses. Finally, we conclude with comments on the physical interpretation of our results in Section 4. For pedagogical completeness, we include, in the appendix, the derivation of the vector polarization tensor. In that calculation, we employ Feynman parametrization and $\epsilon$-regularization in the real time formalism, paying particular attention to the subtleties of Feynman parametrization pointed out by Weldon 


\section{The Scalar Case}

Consider a toy model described by the Lagrangian density,

$$
\mathcal{L}=\mathcal{L}_{0}^{1}\left(\phi_{1}\right)+\mathcal{L}_{0}^{2}\left(\phi_{2}\right)-\frac{\lambda}{2} \phi_{1}^{2} \phi_{2}
$$

where

$$
\mathcal{L}_{0}^{i}=\frac{1}{2} \partial_{\mu} \phi_{i} \partial^{\mu} \phi_{i}-\frac{m_{i}^{2}}{2} \phi_{i}^{2},
$$

with $i=1,2$ (no summation). We ignore the fact that the potential for this theory is unbounded from below, and compute the one-loop contribution to the self-energy of $\phi_{1}$ to demonstrate its analytic structure. The only contribution to the self-energy at one-loop comes from the diagram in fig. 1. One obtains the temperature-dependent part of the self-energy in the usual fashion

$$
\begin{array}{r}
\operatorname{Re} \Sigma^{\beta}\left(p_{0}, \vec{p}\right)=-\lambda^{2} \int \frac{d^{3} k}{(2 \pi)^{3}}\left[\frac{n\left(\omega_{1}\right)}{2 \omega_{1}} \frac{1}{\left(P^{2}-2 \vec{k} \cdot \vec{p}+2 \omega_{1} p_{0}+\Delta m_{12}^{2}\right)}\right. \\
+\frac{n\left(\omega_{2}\right)}{2 \omega_{2}} \frac{1}{\left(P^{2}+2 \vec{k} \cdot \vec{p}+2 \omega_{2} p_{0}-\Delta m_{12}^{2}\right)} \\
\left.+\left(p_{0} \leftrightarrow-p_{0}\right)\right]
\end{array}
$$

where $\omega_{i}^{2}=\vec{k}^{2}+m_{i}^{2}$, for $i=1,2, \Delta m_{12}^{2}=m_{1}^{2}-m_{2}^{2}$, and $n(x)$ is the Bose-Einstein distribution.

The angular integration yields

$$
\operatorname{Re} \Sigma^{\beta}\left(p_{0}, \vec{p}\right)=-\frac{\lambda^{2}}{8 \pi^{2}} \int_{0}^{\infty} d k \frac{k}{p}\left[\frac{n\left(\omega_{1}\right)}{2 \omega_{1}} \ln \left|S_{1}\right|+\frac{n\left(\omega_{2}\right)}{2 \omega_{2}} \ln \left|S_{2}\right|\right],
$$

where

$$
\begin{aligned}
& S_{1}=\frac{\left(p_{0}^{2}-p^{2}+\Delta m_{12}^{2}+2 p k\right)^{2}-4 p_{0}^{2} \omega_{1}^{2}}{\left(p_{0}^{2}-p^{2}+\Delta m_{12}^{2}-2 p k\right)^{2}-4 p_{0}^{2} \omega_{1}^{2}}, \\
& S_{2}=\frac{\left(p_{0}^{2}-p^{2}-\Delta m_{12}^{2}+2 p k\right)^{2}-4 p_{0}^{2} \omega_{2}^{2}}{\left(p_{0}^{2}-p^{2}-\Delta m_{12}^{2}-2 p k\right)^{2}-4 p_{0}^{2} \omega_{2}^{2}} .
\end{aligned}
$$

In order to analyze the behavior of the self-energy close to $P^{\mu}=0$, let us assume $p_{0}=\alpha p$ in (2.4) and take the limit $p \rightarrow 0$. If the result is $\alpha$-dependent, then the value of the double limit will depend on the way $P^{\mu}$ approaches zero and, therefore, would correspond to a non-analytic structure at $P^{\mu}=0$. It is easy to establish that (2.4) in this limit becomes,

$$
\lim _{p \rightarrow 0} \operatorname{Re} \Sigma^{\beta}(\alpha p, \vec{p})=-\frac{\lambda^{2}}{\pi^{2} \Delta m_{12}^{2}} \int_{0}^{\infty} d k k^{2}\left[\frac{n\left(\omega_{1}\right)}{2 \omega_{1}}-\frac{n\left(\omega_{2}\right)}{2 \omega_{2}}\right] .
$$


This limit clearly is independent of $\alpha$, and therefore takes on a unique value showing that the self-energy is analytic at $P^{\mu}=0$. This is also the value that one would have obtained for the self-energy by setting $P^{\mu}=0$ inside the integrand in Eq. (2.3). In such a case, it is clear from (2.3) that $\Delta m_{12}^{2}$ has to be non zero for a well behaved result. At high temperature, the leading contribution to this double limit can be shown to be

$$
\lim _{\substack{p \rightarrow 0 \\ p_{0} \rightarrow 0}} \operatorname{Re} \Sigma^{\beta}\left(p_{0}, p\right) \simeq \frac{\lambda^{2}}{4 \pi} \frac{T}{m_{1}+m_{2}} .
$$

Next, let us turn our attention to a spontaneously broken $\mathrm{U}(1)$ gauge theory.

\section{Abelian Higgs Model}

For simplicity, we will perform the calculation of the polarization tensor for the massive vector boson in the Abelian Higgs model in unitary gauge. Unitary gauge is infamous for complications in the Higgs sector at finite temperature 6 . In the gauge sector, however, these complications are absent and the smaller number of diagrams makes its use preferable for our purposes.

The Lagrangian for the Abelian Higgs model in the unitary gauge is given by

$$
\begin{aligned}
\mathcal{L}= & -\frac{1}{4} F^{\mu \nu} F_{\mu \nu}+\frac{e^{2} v^{2}}{2} A^{\mu} A_{\mu}+\frac{1}{2} \partial^{\mu} \eta \partial_{\mu} \eta-\frac{m^{2}}{2} \eta^{2} \\
& +\frac{e^{2}}{2} A^{\mu} A_{\mu} \eta^{2}+e^{2} v A^{\mu} A_{\mu} \eta-\lambda v \eta^{3}-\frac{\lambda}{4} \eta^{4}
\end{aligned}
$$

where $\eta$ is the Higgs field, $A_{\mu}$ is the $\mathrm{U}(1)$ gauge field and the vacuum expectation value, $v=m / \sqrt{2 \lambda}$. In this gauge, the only one-loop, momentum-dependent correction to the

photon propagator is given by the diagram in fig. 2, which we denote by $\tilde{\Pi}_{\mu \nu}$. This diagram gives,

$$
\begin{aligned}
& \operatorname{Re} \tilde{\Pi}_{00}^{\beta}=4 e^{2} \int \frac{d^{3} k}{(2 \pi)^{3}} {\left[\frac{n\left(\omega_{k}\right)}{2 \omega_{k}} \frac{M^{2}-\left(p_{0}-\omega_{k}\right)^{2}}{\left(p_{0}-\omega_{k}\right)^{2}-\Omega_{k+p}^{2}}+\frac{n\left(\Omega_{k}\right)}{2 \Omega_{k}} \frac{M^{2}-\Omega_{k}^{2}}{\left(p_{0}-\Omega_{k}\right)^{2}-\omega_{k+p}^{2}}\right] } \\
&+\left(p_{0} \rightarrow-p_{0}\right) .
\end{aligned}
$$

Here we have defined $M=e v, \omega_{k}=\sqrt{\vec{k}^{2}+m^{2}}$ and $\Omega_{k}=\sqrt{\vec{k}^{2}+M^{2}}$. (The expression (3.2) is easily obtained by standard techniques. However, because confusion in the literature about the $P^{\mu} \rightarrow 0$ limit is often tied to particular techniques for computing 
finite-temperature diagrams, and since the use of Feynman parametrization in the realtime formalism is a particularly good example of this, we show explicitly in the appendix how to perform such a calculation using this technique, together with regularization of the real-time propagators. For routine calculations, however, Feynman parametrization is impractical.) After doing the angular integration, one obtains

$$
\begin{aligned}
\operatorname{Re} \tilde{\Pi}_{00}^{\beta}\left(p_{0}, p\right)=-\frac{e^{2}}{2 \pi^{2}} \int_{0}^{\infty} d k k & \frac{\left(\Delta m_{12}^{2}+k^{2}+p_{0}^{2}\right) n\left(\omega_{k}\right)}{2 \omega_{k}} \frac{1}{p} \ln \left|S_{1}\right|+\frac{k^{2} n\left(\Omega_{k}\right)}{2 \Omega_{k}} \frac{1}{p} \ln \left|S_{2}\right| \\
& \left.+n\left(\omega_{k}\right) \frac{p_{0}}{p} \ln \left|\frac{\left(p_{0}^{2}-p^{2}+\Delta m_{12}^{2}\right)^{2}-4\left(p_{0} \omega_{k}+p k\right)^{2}}{\left(p_{0}^{2}-p^{2}+\Delta m_{12}^{2}\right)^{2}-4\left(p_{0} \omega_{k}-p k\right)^{2}}\right|\right],
\end{aligned}
$$

where $S_{i}$ are given in (2.5) with $m_{1}=m$ and $m_{2}=M$.

Let us analyze the small- $p^{0}$, small- $p$ behavior of (3.3). For that purpose, let us set as before

$$
p^{0}=\alpha p
$$

Then, for nonzero values of $\Delta m_{12}^{2}=m^{2}-M^{2}$, it is clear that

$$
\lim _{p \rightarrow 0} \operatorname{Re} \tilde{\Pi}_{00}^{\beta}(\alpha p, p)=-\frac{4 e^{2}}{\pi^{2}} \int_{0}^{\infty} d k\left[k^{2} \frac{n\left(\omega_{k}\right)}{2 \omega_{k}}+\frac{k^{4}}{m^{2}-M^{2}}\left(\frac{n\left(\omega_{k}\right)}{2 \omega_{k}}-\frac{n\left(\Omega_{k}\right)}{2 \Omega_{k}}\right)\right]
$$

In particular, this limit is finite, $\alpha$-independent and hence independent of the ratio $p_{0} / p$ as $p_{0}$ and $p$ approach zero. Alternatively, this may be obtained by simply putting $P^{\mu}=0$ in (3.2). So, the double limit is unique, as promised. Furthermore, it is easy to establish that $\operatorname{Re} \tilde{\Pi}_{i i}^{\beta}$ has a unique double limit as well.

The high-temperature limit of (3.5) can be easily obtained to be

$$
\lim _{\substack{p \rightarrow 0 \\ p_{0} \rightarrow 0}} \operatorname{Re} \tilde{\Pi}_{00}^{\beta}\left(p_{0}, p\right)=\frac{1}{2} e^{2} T^{2}
$$

which turns out to be the same as the $\left(p_{0}=0, \vec{p} \rightarrow 0\right)$ limit of the equal mass case $\Delta m_{12}^{2}=0$. (In fact, we note here that even though the expression (3.5) appears to be singular when $m=M$, it indeed has a finite limit as the two masses become degenerate and corresponds to the $p_{0}=0, \vec{p} \rightarrow 0$ limit of the degenerate case. One can, therefore, even foresee using such a mass-splitting regularization in such calculations for the equal mass case.) 


\section{Summary and Physical Implications}

We have shown, both in the context of a scalar toy model as well as for a spontaneously broken Abelian gauge theory, that the finite-temperature one-loop self-energy/polarization tensor at finite temperature has a unique limit as the external four-momentum goes to zero. The absence of the usual non-commuting double limits is traced to the fact that there is (generically) a finite mass difference among the particles propagating in the loop. One can understand this result in the following way. The real part of the one-loop self-energy is related to the imaginary part through the dispersion relation 4 ,

$$
\begin{aligned}
\operatorname{Re} \Sigma_{R}^{\beta}\left(p_{0}, p\right) & =\frac{1}{\pi} \mathcal{P} \int_{-\infty}^{\infty} d u \frac{\operatorname{Im} \Sigma_{R}^{\beta}(u, p)}{u-p_{0}} \\
& =\frac{2}{\pi} \mathcal{P} \int_{0}^{\infty} d u u \frac{\operatorname{Im} \Sigma_{R}^{\beta}(u, p)}{u^{2}-p_{0}^{2}} .
\end{aligned}
$$

The last equality follows from the fact that $\operatorname{Im} \Sigma_{R}^{\beta}\left(p_{0}, p\right)$ is an odd function of $p_{0}$ ] . Here $\Sigma_{R}^{\beta}$ is the retarded two point function related to $\Sigma^{\beta}$ by

$$
\begin{aligned}
& \operatorname{Im} \Sigma_{R}^{\beta}(u, p)=\operatorname{Im} \Sigma^{\beta}(u, p) \tanh \frac{\beta u}{2} \\
& \operatorname{Re} \Sigma_{R}^{\beta}(u, p)=\operatorname{Re} \Sigma^{\beta}(u, p) .
\end{aligned}
$$

As pointed out by Weldon[绝, $\operatorname{Im} \Sigma_{R}^{\beta}(u, p)$ is non-zero only for some values of $u^{2}-p^{2}$. The imaginary part of the self-energy is expressed in terms of the discontinuity of $\Sigma_{R}^{\beta}\left(p_{0}, p\right)$ along these cuts on the real axis,

$$
\lim _{\epsilon \rightarrow 0^{+}}\left(\Sigma_{R}^{\beta}\left(p_{0}+i \epsilon, p\right)-\Sigma_{R}^{\beta}\left(p_{0}-i \epsilon, p\right)\right)=-2 i \operatorname{Im} \Sigma_{R}^{\beta}\left(p_{0}, p\right)
$$

for real $p_{0}$. For fixed $m_{1}$ and $m_{2}$, these cuts exist for

$$
\begin{aligned}
& u^{2}-p^{2} \geq\left(m_{1}+m_{2}\right)^{2}, \\
& u^{2}-p^{2} \leq\left(m_{1}-m_{2}\right)^{2} .
\end{aligned}
$$

The first cut is the usual zero-temperature cut corresponding to the decay of the incoming particle, whereas the second appears only at $T \neq 0$ and represents absorption of a particle from the medium. The first cut does not lend itself to non-commuting double limits, so the only suspect is the second cut. In fact, it is this cut which is responsible for the noncommuting double limits in the case $m_{1}=m_{2}$ 迎. In our case however, the contribution 
of this cut is perfectly well-behaved as $P^{\mu} \rightarrow 0$. In fact, if we denote this contribution by $C_{2}\left(p_{0}, p\right)$, then we obtain

$$
\operatorname{Re} \Sigma_{R}^{\beta}\left(p_{0}, p\right) \ni C_{2}\left(p_{0}, p\right)=\frac{2}{\pi} \mathcal{P} \int_{0}^{\left(p^{2}+\left(m_{1}-m_{2}\right)^{2}\right)^{\frac{1}{2}}} d u u \frac{\operatorname{Im} \Sigma_{R}^{\beta}(u, p)}{u^{2}-p_{0}^{2}}
$$

Performing the change of variables $u \rightarrow u / \sqrt{p^{2}+\left(m_{1}-m_{2}\right)^{2}}$, we obtain

$$
R e \Sigma_{R}^{\beta}\left(p_{0}, p\right) \ni C_{2}\left(p_{0}, p\right)=\frac{2}{\pi} \mathcal{P} \int_{0}^{1} d u u \frac{\operatorname{Im} \Sigma_{R}^{\beta}\left(u \sqrt{p^{2}+\left(m_{1}-m_{2}\right)^{2}}, p\right)}{u^{2}-\frac{p_{0}^{2}}{p^{2}+\left(m_{1}-m_{2}\right)^{2}}}
$$

As long as the masses are different, the zero momentum limit of $C_{2}\left(p_{0}, p\right)$ is well-defined and given by

$$
C_{2}(0,0)=\frac{2}{\pi} \int_{0}^{\left|m_{1}-m_{2}\right|} d u \frac{\operatorname{Im} \Sigma_{R}^{\beta}(u, 0)}{u} .
$$

This limit, however, is not well-defined if the masses are equal. Note that (4.7) is wellbehaved, given that $\operatorname{Im} \Sigma^{\beta}(u, 0)$ is odd in $u$, and goes as $u$ for small $u$.

The results of the previous section might, at first, appear to have far-reaching consequences in the study of finite-temperature field theory, where it has always been assumed that all one-loop self-energies exhibit a non-analytic behavior at vanishing external fourmomentum. One may naturally wonder whether our observation has any effect on standard computations of physical quantities, such as the difference between Debye and plasmon masses in the standard electroweak theory, and whether there could be any effect on studies of the electroweak phase transition [8] In fact it does not, as can be argued in the following way. Our result (3.6) for the $P^{\mu} \rightarrow 0$ limit depends on assuming $p_{0}, p \ll\left|\Delta m^{2}\right| / T$ in (3.2), since (3.2) is dominated by $k \sim T$. However, the region of interest for self-consistently finding the Debye or plasmon poles of the vector propagator is when $p_{0}$ or $p$ take values of order $m_{i} \gg\left|\Delta m^{2}\right| / T$. In that regime, $\Delta m_{12}^{2}$ can be ignored in (3.3), in which case one recovers the usual non-commuting double limits. The qualitative features of our results are shown in fig. 3. For $p_{0}$ and $p$ small compared to $\left|\Delta m^{2}\right| / T$, the functions $\Pi_{00}^{\beta}\left(p_{0}, 0\right)$ and $\Pi_{00}^{\beta}(0, p)$ tend to the same limit. However, at order $m$, the functions take on different values. As the mass difference goes to zero, it is clear that the unique limit disappears, as well. 


\section{Appendix A. Polarization Tensor Calculation}

In unitary gauge, the only one-loop momentum-dependent correction to the photon propagator is given by fig. 2 . We denote this $\tilde{\Pi}_{\mu \nu}$. We will compute this contribution in the real-time formalism, using Feynman parametrization, and $\epsilon$-regularization of the propagators. We obtain a regulated expression,

$$
\begin{aligned}
& \tilde{\Pi}_{\mu \nu}\left(p_{0}, p\right)=4 i e^{2} M^{2} \int \frac{d^{4} k}{(2 \pi)^{4}}\left(g_{\mu \nu}-\frac{k_{\mu} k_{\nu}}{M^{2}}\right)\left[\mathcal{P}_{\epsilon}\left(\frac{1}{D_{1}}\right)-2 \pi i\left(n\left(\left|k_{0}\right|\right)+\frac{1}{2}\right) \delta_{\epsilon}\left(D_{1}\right)\right] \\
& {\left[\mathcal{P}_{\epsilon}\left(\frac{1}{D_{2}}\right)-2 \pi i\left(n\left(\left|p_{0}+k_{0}\right|\right)+\frac{1}{2}\right) \delta_{\epsilon}\left(D_{2}\right)\right], }
\end{aligned}
$$

where $D_{1}=K^{2}-M^{2}$ and $D_{2}=(K+P)^{2}-m^{2}$, while the regulated principal value and $\delta$-function are defined by

$$
\begin{aligned}
\delta_{\epsilon}(x) & =\frac{1}{\pi} \frac{\epsilon}{x^{2}+\epsilon^{2}}, \\
\mathcal{P}_{\epsilon}\left(\frac{1}{x}\right) & =\frac{x}{x^{2}+\epsilon^{2}} .
\end{aligned}
$$

The real part of the $T \neq 0$ contribution is

$$
\begin{array}{r}
\operatorname{Re} \tilde{\Pi}_{\mu \nu}^{\beta}=4 e^{2} \int \frac{d^{4} k}{(2 \pi)^{3}}\left(M^{2} g_{\mu \nu}-k_{\mu} k_{\nu}\right)\left[\mathcal{P}_{\epsilon}\left(\frac{1}{K^{2}-M^{2}}\right) n\left(\left|p_{0}+k_{0}\right|\right) \delta_{\epsilon}\left((P+K)^{2}-m^{2}\right)\right. \\
\left.+\mathcal{P}_{\epsilon}\left(\frac{1}{(P+K)^{2}-m^{2}}\right) n\left(\left|k_{0}\right|\right) \delta_{\epsilon}\left(K^{2}-M^{2}\right)\right]
\end{array}
$$

There is an implicit limit of $\epsilon \rightarrow 0$ in all the above expressions. This limit is to be taken at the end, after all relevant integrations.

Let us note that the $\epsilon$-regularization is superfluous for generic values of the vector and scalar masses. In the case, however, where $M=m$, the conventional calculation becomes ambiguous at $P^{\mu}=0$. So, we will perform the calculation for an arbitrary infinitesimal $\epsilon$ and we will let $\epsilon$ go to zero at the end.

Define

$$
h_{n}=\int d k_{0} k_{0}^{n} n\left(\left|k_{0}\right|\right) \mathcal{P}_{\epsilon}\left(\frac{1}{(P+K)^{2}-m^{2}}\right) \delta_{\epsilon}\left(K^{2}-M^{2}\right)
$$

and

$$
\tilde{h}_{n}=\int d k_{0} k_{0}^{n} n\left(\left|k_{0}\right|\right) \mathcal{P}_{\epsilon}\left(\frac{1}{(P+K)^{2}-M^{2}}\right) \delta_{\epsilon}\left(K^{2}-m^{2}\right) .
$$


In terms of these functions, the polarization tensor becomes

$$
\operatorname{Re} \tilde{\Pi}_{00}^{\beta}=4 e^{2} \int \frac{d^{3} k}{(2 \pi)^{3}}\left[M^{2} h_{0}-h_{2}+M^{2} \tilde{h}_{0}-\tilde{h}_{2}-2 p_{0} \tilde{h}_{1}-p_{0}^{2} \tilde{h}_{0}\right] .
$$

The other components have similar expressions. Our task is to compute the functions $h_{n}$. Let us rewrite (A.4) as

$$
\begin{aligned}
h_{n} & =\frac{1}{\pi} \int_{-\infty}^{\infty} d k_{0} \frac{k_{0}^{n}}{e^{\beta\left|k_{0}\right|}-1} \frac{\epsilon}{\left(k_{0}^{2}-\Omega_{k}^{2}\right)^{2}+\epsilon^{2}} \frac{\left(p_{0}+k_{0}\right)^{2}-\omega_{p+k}^{2}}{\left(\left(p_{0}+k_{0}\right)^{2}-\omega_{p+k}^{2}\right)^{2}+\epsilon^{2}} \\
& =\frac{1}{\pi} \int_{0}^{\infty} \frac{d k_{0} k_{0}^{n}}{e^{\beta k_{0}}-1} \frac{\epsilon}{\left.\left(k_{0}^{2}-\Omega_{k}^{2}\right)^{2}+\epsilon^{2}\right)} \frac{\left(p_{0}+k_{0}\right)^{2}-\omega_{p+k}^{2}}{\left(\left(p_{0}+k_{0}\right)^{2}-\omega_{p+k}^{2}\right)^{2}+\epsilon^{2}}+(-1)^{n}\left(p_{0} \rightarrow-p_{0}\right) \\
& \equiv H_{n}\left(p_{0}, p\right)+(-1)^{n} H_{n}\left(-p_{0}, p\right) .
\end{aligned}
$$

These integrals can now be calculated using the contour shown in fig. 4. Clearly, the integrals vanish along the arc. However, since the Bose-Einstein distribution has a series of poles alond the imaginary axis, the integration along this axis would appear to give a non-vanishing contribution. It is easy to establish, however, that in the limit $\epsilon \rightarrow 0$, the term $\delta_{\epsilon}$ would regulate this contribution to zero. We proceed by expressing each fraction in the integrand as the sum or difference of propagators with different analytic properties (i.e. differing in their $i \epsilon$ prescriptions), to obtain

$$
H_{n}\left(p_{0}, p\right)=\frac{i}{4 \pi} \int_{0}^{\infty} \frac{d k_{0} k_{0}^{n}}{e^{\beta k_{0}}-1} \sum_{a, b= \pm 1} b \frac{1}{\left(p_{0}+k_{0}\right)^{2}-\omega_{p+k}^{2}+a i \epsilon} \frac{1}{k_{0}^{2}-\Omega_{k}^{2}+b i \epsilon} .
$$

Next, we combine denominators using Feynman parametrization, appropriately modified by Weldon, [4], and Bedaque and Das [9],

$$
\frac{1}{A+i \alpha \epsilon} \frac{1}{B+i \beta \epsilon}=P \int_{0}^{1} \frac{d x}{[x(A+i \alpha \epsilon)+(1-x)(B+i \beta \epsilon)]^{2}}+2 \pi i \frac{(\alpha-\beta) \delta(\beta A-\alpha B)}{A-B+i(\alpha-\beta) \epsilon} .
$$

We shall denote the contribution to $H_{n}$ from the integral over the Feynman parameter by $H_{n}^{x}$ and the contribution from the delta-function by $H_{n}^{\delta}$.

$$
\begin{gathered}
H_{n}^{x}\left(p_{0}, p\right)=\frac{i}{4 \pi} \int_{0}^{\infty} d k_{0} \frac{k_{0}^{n}}{e^{\beta k_{0}}-1} \sum_{a, b= \pm 1} b \int_{0}^{1} d x \frac{1}{\left[\left(k_{0}+x p_{0}\right)^{2}-\phi_{k}^{2}+(x a+(1-x) b) i \epsilon\right]^{2}} \\
=\frac{i}{4 \pi}\left\{\frac { \partial } { \partial \epsilon } \int _ { 0 } ^ { \infty } d k _ { 0 } \frac { k _ { 0 } ^ { n } } { e ^ { \beta k _ { 0 } } - 1 } \sum _ { a , b = \pm 1 } b \int _ { 0 } ^ { 1 } d x \frac { i } { y } \left[\frac{1}{k_{0}+x p_{0}-\phi_{k}+y \frac{i \epsilon}{2 \phi_{k}}} .\right.\right. \\
\left.\left.\frac{1}{k_{0}+x p_{0}+\phi_{k}-y \frac{i \epsilon}{2 \phi_{k}}}\right]\right\}_{\epsilon=0},
\end{gathered}
$$


where

$$
\phi_{k}=\left(-x(1-x) p_{0}^{2}+(1-x) \Omega_{k}^{2}+x \omega_{p+k}^{2}\right)^{\frac{1}{2}}
$$

and

$$
y=x(a-b)+b
$$

We are now able to do the $k_{0}$-integration by picking the poles in the first quadrant,

$$
\begin{aligned}
H_{n}^{x}\left(p_{0}, p\right)=-\frac{i}{2}\left\{\frac{\partial}{\partial \epsilon}[\right. & \int_{0}^{1} d x \frac{1}{2 \phi_{k}+\frac{i \epsilon}{\phi_{k}}} \frac{\left(\phi_{k}-x p_{0}+\frac{i \epsilon}{2 \phi_{k}}\right)^{n}}{\beta\left(\phi_{k}-x p_{0}+\frac{i \epsilon}{2 \phi_{k}}\right)}-1 \\
+ & \int_{0}^{\frac{1}{2}} \frac{d x}{1-2 x} \frac{1}{2 \phi_{k}+(1-2 x) \frac{i \epsilon}{\phi_{k}}} \frac{\left(\phi_{k}-x p_{0}+(1-2 x) \frac{i \epsilon}{2 \phi_{k}}\right)^{n}}{e^{\beta\left(\phi_{k}-x p_{0}+(1-2 x) \frac{i \epsilon}{2 \phi_{k}}\right)}-1} \\
& \left.\left.\int_{\frac{1}{2}}^{1} \frac{d x}{1-2 x} \frac{1}{2 \phi_{k}-(1-2 x) \frac{i \epsilon}{\phi_{k}}} \frac{\left(\phi_{k}-x p_{0}-(1-2 x) \frac{i \epsilon}{2 \phi_{k}}\right)^{n}}{e^{\beta\left(\phi_{k}-x p_{0}-(1-2 x) \frac{i \epsilon}{2 \phi_{k}}\right)}-1}\right]\right\}_{\epsilon=0} .
\end{aligned}
$$

Upon defining a new variable

$$
z_{k}(\alpha)=\left(-x(1-x) p_{0}^{2}+(1-x) \Omega_{k}^{2}+x \omega_{p+k}^{2}+\alpha\right)^{\frac{1}{2}}, \quad z_{k}(0)=\phi_{k}
$$

Eq. (A.13) reduces to

$$
H_{n}^{x}\left(p_{0}, p\right)=\frac{1}{2}\left\{\frac{\partial}{\partial \alpha} \int_{0}^{\frac{1}{2}} \frac{d x}{z_{k}} \frac{\left(z_{k}-x p_{0}\right)^{n}}{e^{\beta\left(z_{k}-x p_{0}\right)}-1}\right\}_{\alpha=0} .
$$

The term involving the delta-function in the Feynman parametrization formula can be easily computed to contribute

$$
H_{n}^{\delta}\left(p_{0}, p\right)=-\frac{1}{2 R} \frac{\left(-p_{0} / 2+R\right)^{n}}{e^{\beta\left(-p_{0} / 2+R\right)}-1} \frac{1}{\omega_{p+k}^{2}-\Omega_{k}^{2}-2 p_{0} R}
$$

where

$$
R=\sqrt{-\frac{p_{0}^{2}}{4}+\frac{1}{2}\left(\omega_{p+k}^{2}+\Omega_{k}^{2}\right)} .
$$

The function $\tilde{h}_{n}$, defined by (A.5) can be calculated in the same fashion.

Recalling (A.6), and collecting only the Feynman parameter dependent terms, we obtain

$$
\begin{aligned}
\operatorname{Re} \tilde{\Pi}_{00}^{\beta} \ni 2 e^{2}\left\{\frac{\partial}{\partial \alpha} \int \frac{d^{3} k}{(2 \pi)^{3}} \int_{0}^{\frac{1}{2}}\right. & d x\left(M^{2}-\left(z_{k}+x p_{0}\right)^{2}\right) \frac{n\left(z_{k}+x p_{0}\right)}{z_{k}} \\
& \left.+\left(M^{2}-\left(\tilde{z}_{k}+(1-x) p_{0}\right)^{2}\right) \frac{n\left(\tilde{z}_{k}-x p_{0}\right)}{\tilde{z}_{k}}\right\}_{\alpha=0} \\
& +\left(p_{0} \rightarrow-p_{0}\right)
\end{aligned}
$$


where $\tilde{z}_{k} \leftrightarrow z_{k}$ as $m \leftrightarrow M$. The $x$-integration can be performed after a change of variables

$$
\begin{aligned}
& x \rightarrow 1-x \\
& \vec{k} \rightarrow-\vec{k}-\vec{p},
\end{aligned}
$$

in the second term, and

$$
w=z_{k}+x p_{0},
$$

in the resulting integrand. All the dependence on $\alpha$ resides now in the limits of integration. Taking the derivative with respect to $\alpha$ and the limit $\alpha \rightarrow 0$ yields,

$$
\begin{aligned}
\operatorname{Re} \tilde{\Pi}_{00}^{\beta} \ni 2 e^{2} \int \frac{d^{3} k}{(2 \pi)^{3}} & {\left[\frac{n\left(\omega_{k}\right)}{\omega_{k}} \frac{M^{2}-\left(p_{0}-\omega_{k}\right)^{2}}{\left(p_{0}-\omega_{k}\right)^{2}-\Omega_{k+p}^{2}}+\frac{n\left(\Omega_{k}\right)}{\Omega_{k}} \frac{M^{2}-\Omega_{k}^{2}}{\left(p_{0}-\Omega_{k}\right)^{2}-\omega_{k+p}^{2}}\right.} \\
+ & \left.\frac{1}{R} \frac{M^{2}-\frac{1}{2}\left(\Omega_{k}^{2}+\omega_{p+k}^{2}\right)-p_{0} R}{\omega_{p+k}^{2}-\Omega_{k}^{2}+2 p_{0} R}\left[\frac{1}{e^{\beta\left(\frac{p_{0}}{2}+R\right)}-1}-\frac{1}{e^{\beta\left(-\frac{p_{0}}{2}+R\right)}-1}\right]\right] \\
& +\left(p_{0} \rightarrow-p_{0}\right),
\end{aligned}
$$

where $R$ is given by Eq. (A.17). If the delta function contribution to the Feynman parametrization formula (A.9) were incorrectly left out, then (A.21) would be the complete expression for $\operatorname{Re} \tilde{\Pi}_{00}^{\beta}$. It is interesting to note that in such a case (A.21) would agree with the expression derived within the imaginary-time formalism for $p_{0}=2 \pi i \ell T$, since then the third term would vanish identically. However, (A.21) as it stands is the wrong analytic continuation of the euclidean expression. Indeed, the contribution of the delta function (A.16) exactly cancels the third term in (A.21) and, therefore, the complete expression is

$$
\begin{aligned}
& \operatorname{Re} \tilde{\Pi}_{00}^{\beta}=4 e^{2} \int \frac{d^{3} k}{(2 \pi)^{3}} {\left[\frac{n\left(\omega_{k}\right)}{2 \omega_{k}} \frac{M^{2}-\left(p_{0}-\omega_{k}\right)^{2}}{\left(p_{0}-\omega_{k}\right)^{2}-\Omega_{k+p}^{2}}+\frac{n\left(\Omega_{k}\right)}{2 \Omega_{k}} \frac{M^{2}-\Omega_{k}^{2}}{\left(p_{0}-\Omega_{k}\right)^{2}-\omega_{k+p}^{2}}\right] } \\
&+\left(p_{0} \rightarrow-p_{0}\right),
\end{aligned}
$$

which agrees with the imaginary-time expression for real $p_{0}$.

P.A. was supported by DOE grant DE-FG06-91ER40614. P.B. was supported in part by DOE grant DE-FG02-91ER40685 and in part by CAPES. A.D. was supported in part by DOE grant DE-FG02-91ER40685. A.D. also thanks the Division of Educational Programs of Argonne National Laboratory. S.V. was supported by DOE grants DE-FG06-91ER40614 and W-31-109-ENG-38. S.V. acknowledges useful discussions with G. Bodwin, L. Brown, I. Knowles, L. Yaffe, and C. Zachos and thanks U. Sarid for his Feynman diagram drawing code. 


\section{References}

[1] V. P. Silin, Sov. Phys. JETP 11, 1136 (1960);

D.J. Gross, R.D. Pisarski and L.G. Yaffe, Rev. Mod. Phys. 53, 43 (1981);

V.V. Klimov, Sov. Phys. JETP 55, 199 (1982);

H.A. Weldon, Phys. Rev. D26, 1394 (1982);

E. Braaten and R. D. Pisarski, Nucl. Phys. B337, 569 (1990) and B339, 310 (1990),

Phys. Rev. Lett. 64, 1338 (1990), Phys. Rev. D45, 1827 (1992);

J. Frenkel and J. C. Taylor, Nucl. Phys. B334, 199 (1990) and B374, 156 (1992).

[2] Y. Fujimoto and H. Yamada, Z. Phys. C37, 265 (1988);

P. S. Gribosky and B. R. Holstein, Z. Phys. C47, 205 (1990);

P. Bedaque and A. Das, Phys. Rev. D45, 2906 (1992)

[3] H. A. Weldon, Phys. Rev. D28, 2007 (1983). Our expression (4.4b) for the $T \neq 0$ cut disagrees with Weldon's corresponding cut.

[4] H. A. Weldon, West Virginia University Preprint WVU-992 (August 1992).

[5] The reader wishing an introduction to perturbation theory at finite temperature may try J.I. Kapusta, "Finite-temperature field theory" Cambridge Univ. Press, Cambridge, 1989.

[6] P. Arnold, E. Braaten, and S. Vokos, Phys. Rev. D46, 3576 (1992).

[7] A. A. Abrikosov, L. P. Gorkov and I. E. Dzyaloshinski, "Methods of Quantum Field Theory in Statistical Physics", Dover, New York, 1963;

A. L. Fetter and J. D. Walecka, "Quantum Theory of Many Particle Systems", McGraw-Hill, New York, 1971;

H. Umezawa, H. Matsumoto, and M. Tachiki, "Thermo Field Dynamics and Condensed States", North-Holland, Amsterdam, 1982;

S. Jeon, Univ. of Washington Preprint UW/PT-92-03.

[8] M. Carrington, Phys. Rev. D45, 2933 (1992);

M. Dine, G. Leigh, P. Huet, A. Linde, and D. Linde, Phys. Rev. D46, 550 (1992), and Phys. Lett. B283, 319 (1992);

G. Boyd, D. Brahm, S. Hsu, Enrico Fermi Institute Preprint EFI-92-22 (1992);

P. Arnold and O. Espinosa, Univ. of Washington Preprint UW/PT-92-18.

[9] P. Bedaque and A. Das, Rochester Preprint UR 1275, ER-40685-727 (September 1992). 


\section{Figure Captions}

Fig. 1. The one-loop contribution to the $\phi_{1}$ self-energy.

Fig. 2. The only one-loop, momentum-dependent contribution to the vector self-energy, in unitary gauge.

Fig. 3. For $p_{0}$ and $p$ small compared to $\left|\Delta m^{2}\right| / T$, the functions $\Pi_{00}^{\beta}\left(p_{0}, 0\right)$ and $\Pi_{00}^{\beta}(0, p)$ tend to the same limit. However, at order $m$, the functions take on different values. As the mass difference goes to zero, it is clear that the unique limit disappears, as well.

Fig. 4. Contour in the complex $k^{0}$-plane used in the integration. The limit $\epsilon^{\prime} \rightarrow 0$ is implied. 


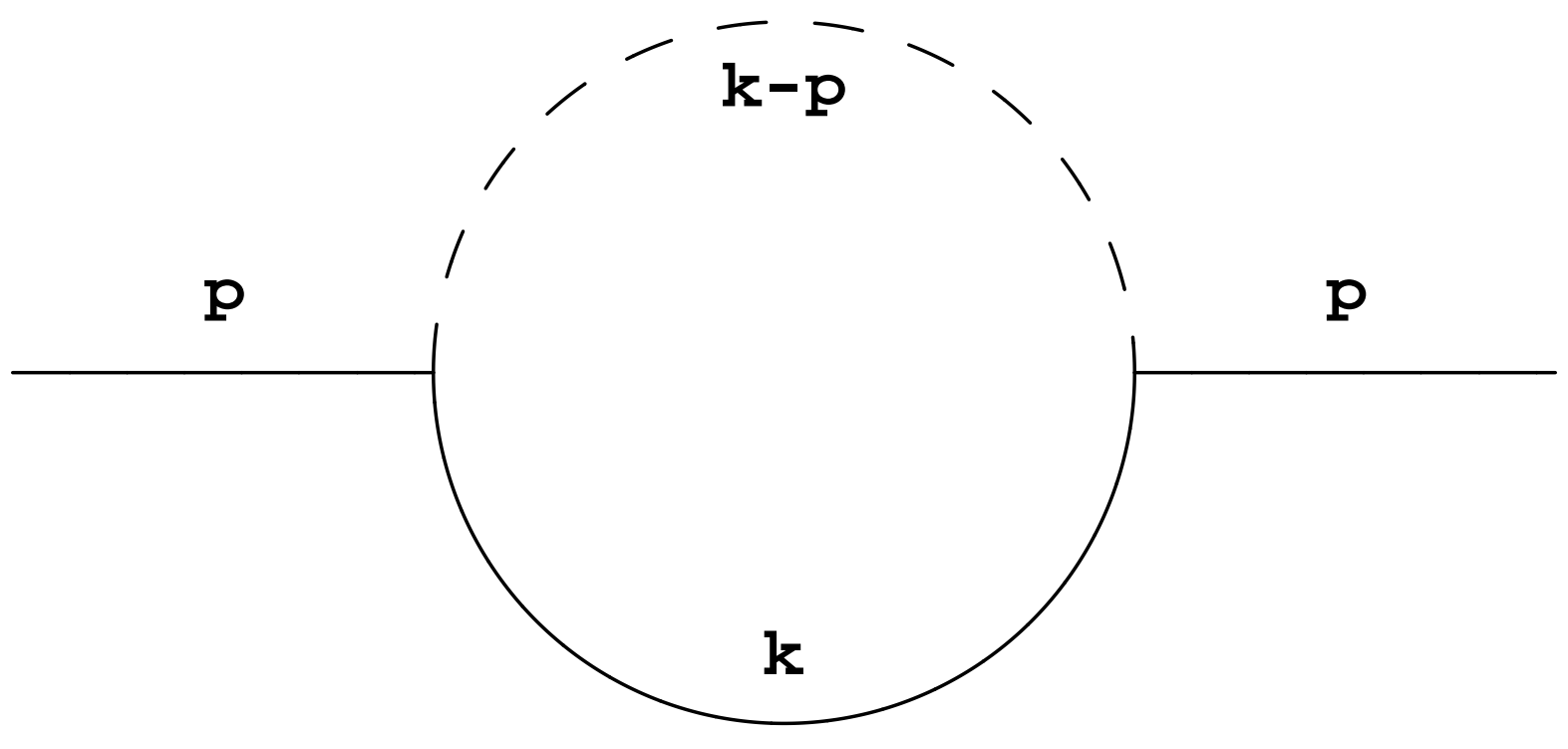

Fig. 1 


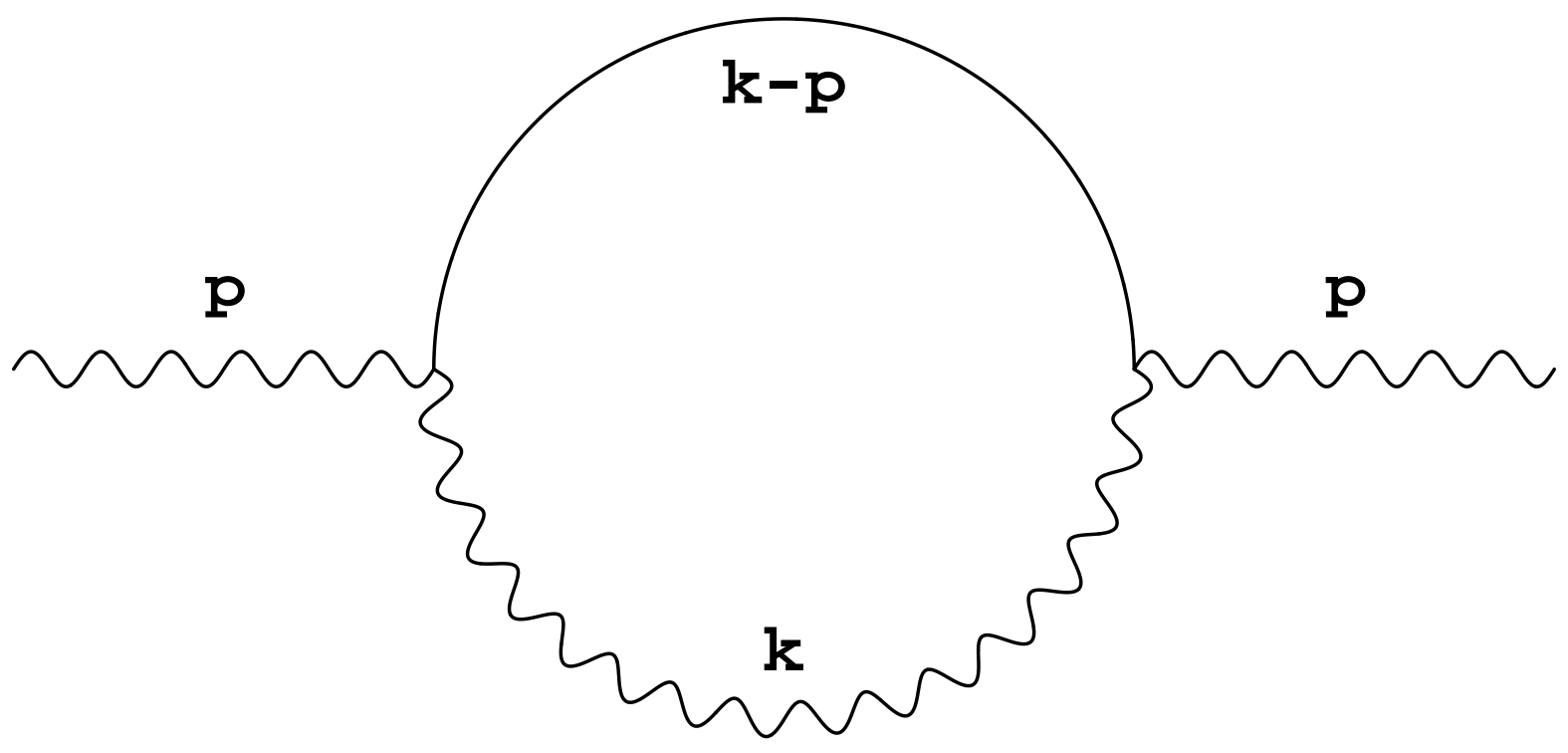

Fig. 2 
Fig. 3

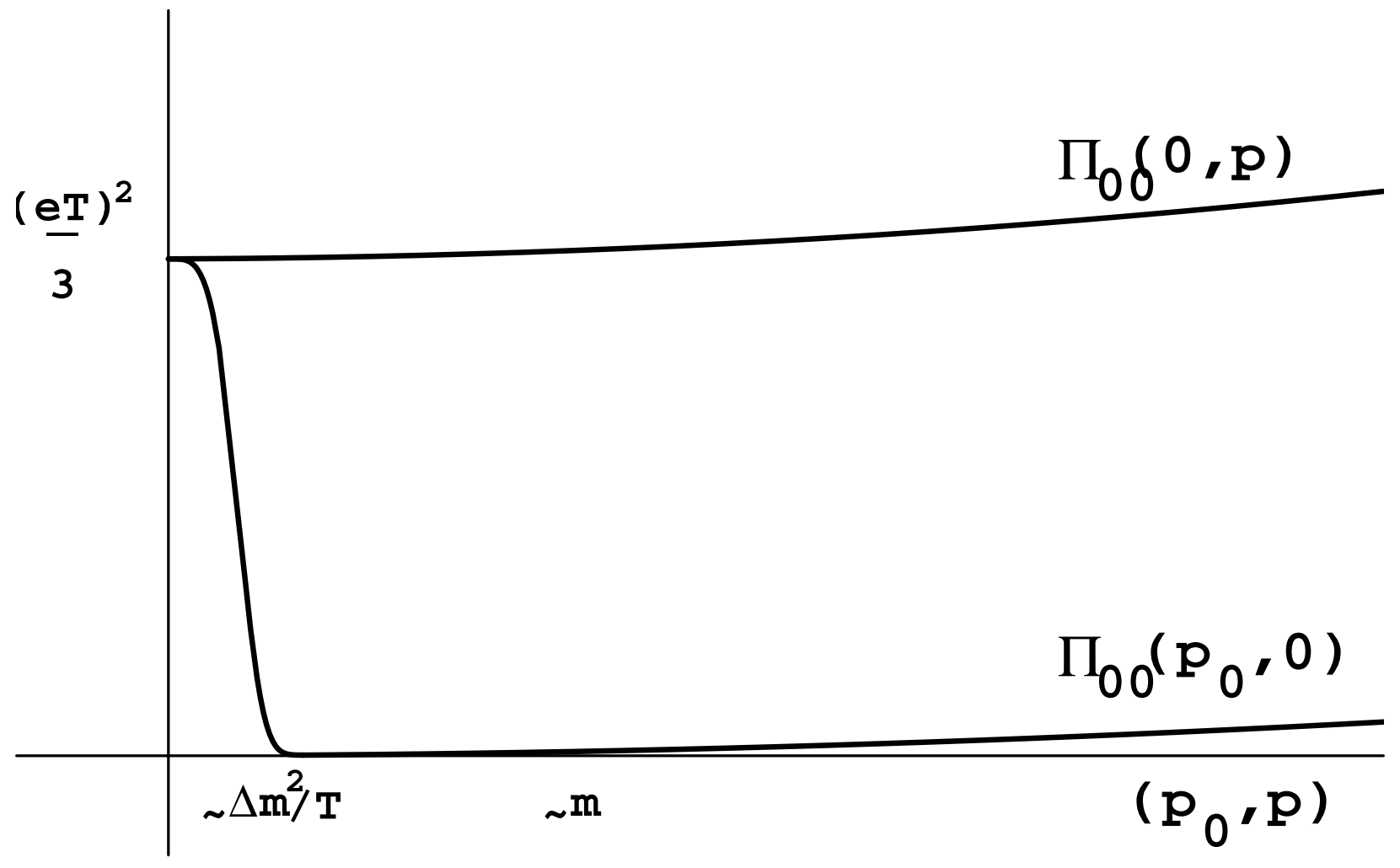




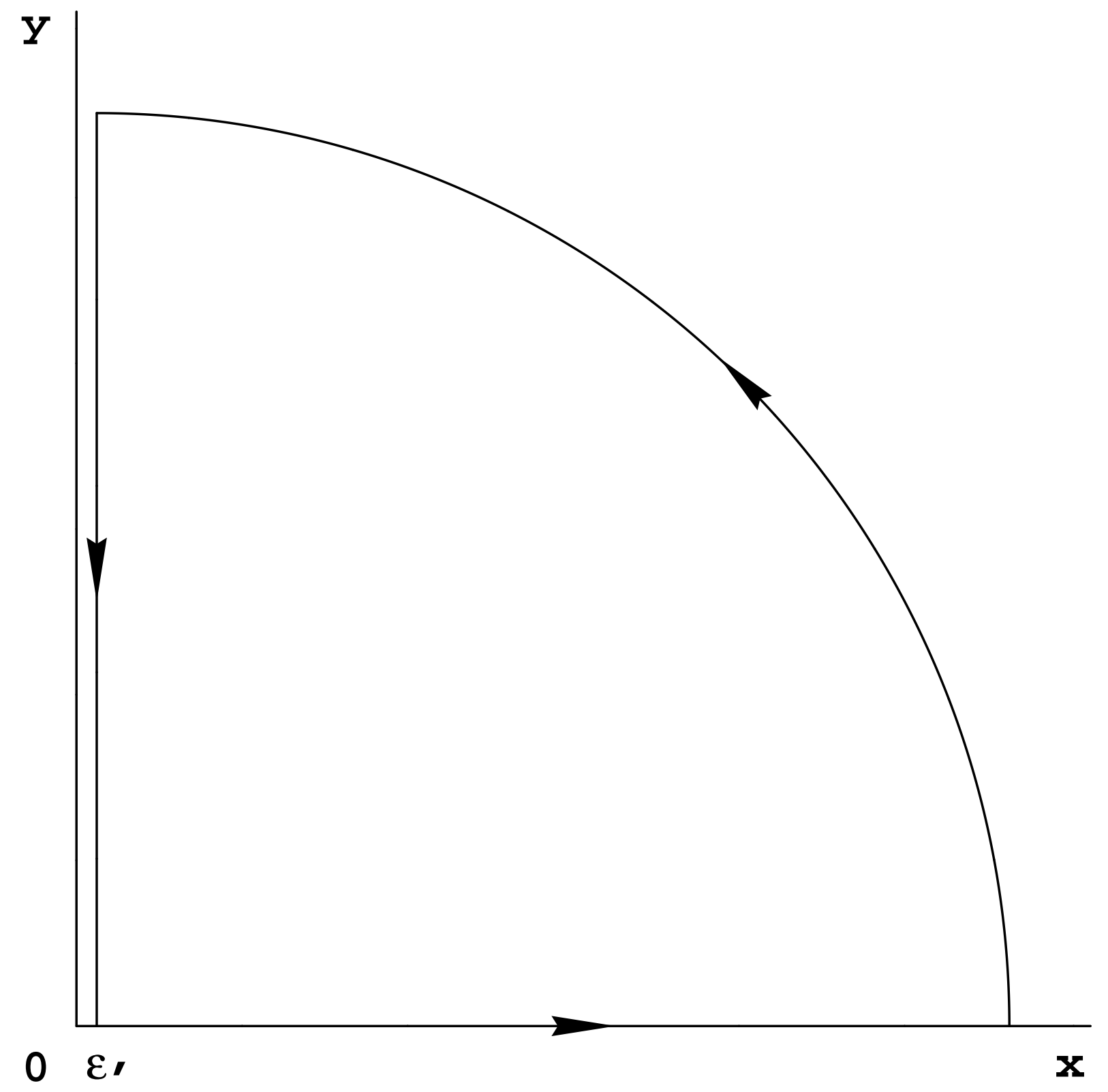

Fig. 4 